\title{
THE FIFTEENTH REGULAR MEETING OF THE SOUTHWESTERN SECTION
}

The fifteenth regular meeting of the Southwestern Section of this Society was held at the University of Kansas on Saturday, December 2, 1922. The total attendance was twenty-eight, including the following sixteen members:

Ashton, Black, Candy, Garrett, Ingold, Kendall, Lefschetz, J. V. McKelvey, U. G. Mitchell, R. L. Moore, Roever, Edwin R. Smith, G. W. Smith, Stouffer, J. S. Turner, Wheeler.

Professor Ashton occupied the chair, being relieved during the afternoon session by Professor Lefschetz. The morning session and part of the afternoon session were devoted to the reading of the papers listed below. During the afternoon session Professor R. L. Moore gave a special lecture by invitation on Continuous curves from the view-point of analysis situs. This paper will appear in full in a later number of this Bulletin. It was voted to hold the next meeting of the Southwestern Section at the University of Missouri. The following program committee was elected: Professors Hedrick (chairman), McKelvey, and Stouffer (secretary).

The titles and abstracts of the papers read follow below.

1. Professor A. L. Candy: Cyclic operations on determinants.

This paper consists chiefly of three theorems: (I) If the rows of a given determinant $\Delta$ be added cyclically $s$ in a set, the resulting determinant $\Delta_{s}$ will be equal to zero, or $s \cdot \Delta$, according as $n$ (order of $\Delta$ ) and $s$ are commensurable, or incommensurable. (II) If the elements in each column of a determinant $\Delta$ be permuted cyclically in all possible ways, and the $n^{n}$ determinants thus formed be collected into groups such that each determinant in the group shall have the same subscripts in the first row, the sum of the determinants in any one of these groups will be equal to $\pm \lambda \Delta$, or zero, according as the weight of the group (the sum of the subscripts in any first row) is, or is not, a multiple of $n$, where $\lambda$ is $1, n$, a submultiple of $n$, or the sum of two or more such numbers. (III) The sum of the $n$ ! determinants that can be formed by making the $n$ ! permutations of the columns of a circulant is equal to zero.

2. Professors Claribel Kendall and G. W. Smith: On the conditions for associativity in linear algebra. 
In a linear algebra of $n$ units the differences $\left(e_{i} e_{j}\right) e_{k}-e_{i}\left(e_{j} e_{k}\right)$ lead to $n^{4}$ quadratic forms in the $n^{3}$ constants of multiplication. The authors establish certain relations among these forms, and in the case $n=2$ determine eight relations among them.

3. Professor G. W. Smith: Analytic expression for line segments.

In this paper the author sets up an analytic expression which represents $f(x)$ in a certain interval $a<x<b(a>0)$ and only in this interval, the expression being imaginary for other values of $x$. By a combination of these he represents analytically such geometric figures as triangles and squares.

4. Professor W. H. Roever: Singularity in the boundary curve of the projection of a surface.

In the boundary curve of the projection of a surface there may be points at which the curve stops abruptly. This is illustrated in the case of the orthographic projection of a torus, for which the curve in question consists of two parallels to an ellipse. If the torus is sufficiently inclined to the plane of projection the inner parallel has four cusps, and only that portion of it which lies between a particular pair of these cusps is visible and thus the inner boundary apparently stops at these points. Several methods of determining such singularities.

5. Professor Edwin R. Smith: The determination of hidden periodicities by difference methods.

If $y=f\left(x, \alpha_{1}, \alpha_{2}, \alpha_{3}, \cdots, \alpha_{n}\right)$ is a function of $x$ with $n$ distinct simple unknown periods $\alpha_{1}, \alpha_{2}, \alpha_{3}, \cdots, \alpha_{n}$, it is shown that the function satisfies a difference equation of the form $u(x)-C_{1} u_{n-1}(x)-C_{2} u_{n-2}(x)-\cdots-(-1)^{n} C_{n} u_{1}(x)=0$, where $C_{1}, C_{2}, C_{3}, \cdots, C_{n}$ are symmetric functions of $\cos \alpha_{1}$, $\cos \alpha_{2}, \cdots, \cos \alpha_{n}$ and $u_{0}(x)=y_{x+1}-y_{x}, u_{i}(x)=u_{i-1}(x-1)$ $-u_{i-1}(x+1),(i=1,2, \cdots, n)$. The values of $C_{1}, C_{2}, C_{3}$, $\cdots, C_{n}$ corresponding to a given set of observations may be found by any other convenient method. The method of least squares leads to results similar to those obtained by the Lagrange-Dale method.

6. Professor S. Lefschetz: Concerning Nöther's theorem on curves traced on non-singular surfaces.

The theorem in question states that all algebraic curves traced on a generic surface of order $m>3$ in ordinary space are complete intersections. 
In his Bordin prize paper the author gave the first complete proof of this important proposition, or rather of a more general one which includes it as a special case. One of the main features of the proof consists in showing that not all double integrals of the first kind attached to the surfaces in question have all periods equal to zero. In this paper a notable simplification of this part of the proof is given, applicable when it is desired to prove strictly Nöther's theorem and no more. It consists essentially in building up a surface of order $m$ which has an almost obvious integral with a non-zero period.

7. Professors E. R. Hedrick and Louis Ingold: Remarks concerning the Michelson-Morley experiment.

Because of prevailing differences of opinion relating to the celebrated Michelson-Morley experiment a new investigation of the mathematical theory seems to be desirable. The work confirms, by an independent calculation, certain results obtained by Righi, and this is of great importance since the accuracy of his work has been called in question (OBSERvaTORY, vol. 44 (1921), pp. 340-341).

8. Professor J. S. Turner: Extensions of the Euler and Seelhoff determinants, with examples of their use in determining whether a number is prime.

It is first pointed out that the non-simple reduced forms of the odd Euler determinants become simple forms, i.e., forms containing no product term when multiplied by 2 . Hence if a prime $p$ has the quadratic character of a non-simple reduced form, then $2 p$ has a single representation by a simple form. A list of negative determinants follows in which there are three reduced forms in each genus, such that four times each non-simple form is a simple form. If $p$ has the quadratic character of a simple form, $4 p$ has a single representation by that form. Seelhoff's list (AMERICAN Journal, vols. 7-8) of determinants in which certain genera contain only simple reduced forms is extended. A list of determinants follows in which certain genera contain only ambiguous reduced forms, i.e., forms which are simple or become simple on multiplication by 2 . The final list contains determinants for which all the forms in certain genera are simple or become simple on multiplication by 4 . The advantages of the new determinants are explained and illustrated by examples. These examples also illustrate an improved method of exclusion.

E. B. Stouffer, Secretary of the Section. 